\title{
$L^{2}$ ASYMPTOTES FOR THE KLEIN-GORDON EQUATION
}

\section{STUART NELSON}

ABSTRACT. An approximation $a(x, t)$ is obtained for solutions $u(x, t)$ of the Klein-Gordon equation. $a(x, t)$ can be expressed in terms of the Fourier transforms of the Cauchy data and it is shown that $\|a(\cdot, t)-u(\cdot, t)\|_{2 \rightarrow 0}$ as $t \rightarrow \infty$. This result is applied to show how energy distributes among various conical regions.

A wide class of solutions to the Klein-Gordon equation

$$
\sum_{i=1}^{n} \frac{\partial^{2} u}{\partial x_{i}^{2}}-\frac{\partial^{2} u}{\partial t^{2}}=u
$$

can be written in the form

$$
\begin{array}{r}
u(x, t)=(2 \pi)^{-n / 2} \int e^{i x \cdot y}\left[F(y) \cos t \sqrt{ }\left(1+y^{2}\right)\right. \\
\left.+G(y) \sin t \sqrt{ }\left(1+y^{2}\right)\right] d^{n} y \\
=(2 \pi)^{-n / 2} \int e^{i x \cdot y\left[\psi(y) \exp \left[i t \sqrt{ }\left(1+y^{2}\right)\right]\right.} \\
\left.+\phi(y) \exp \left[-i t \sqrt{ }\left(1+y^{2}\right)\right]\right] d^{n} y
\end{array}
$$

where $F=\psi+\phi, G=i(\psi-\phi)$ are in $L^{2}\left(R^{n}\right)$ and the integral over $R^{n}$ is interpreted in the sense of Plancherel's theorem. Our main result is

TheOREM 1. Define $a(x, t)=0$ for $|x|>t$ and for $|x|<t$ define

$$
\begin{gathered}
a(x, t)=\left\{e^{i \theta(x, t)} \psi\left(\frac{-x}{\sqrt{ }\left(t^{2}-x^{2}\right)}\right)+e^{-i \theta(x, t)} \phi\left(\frac{x}{\sqrt{ }\left(t^{2}-x^{2}\right)}\right)\right\} \rho(x, t), \\
\theta(x, t) \equiv n \pi / 4+\sqrt{ }\left(t^{2}-x^{2}\right), \quad \rho(x, t) \equiv t\left(t^{2}-x^{2}\right)^{-(n+2) / 4}
\end{gathered}
$$

where $\psi$ and $\phi$ are the same $L^{2}$ functions as in (1). Then

$$
\|u(\cdot, t)-a(\cdot, t)\|_{2}^{2} \equiv \int|u(x, t)-a(x, t)|^{2} d^{n} x \rightarrow 0
$$

as $t \rightarrow \infty$.

Received by the editors March 16, 1970.

AMS 1968 subject classifications. Primary 3516, 3576; Secondary 4240, 8135, 8335.

Key words and phrases. Klein-Gordon equation, Cauchy problem, asymptotic behavior, $L^{\infty}$ decay, $L^{2}$ approximation, energy in conical region, Virial theorem, Riemann-Lebesgue theorem. 
Before starting on the proof of Theorem 1 we mention a corollary. Define

$$
\begin{aligned}
\chi(x, t) & =1 & & \text { if }|x|<t \\
& =0 & & \text { otherwise. }
\end{aligned}
$$

Corollary 1.

$$
\lim _{t \rightarrow \infty}\|\chi u(\cdot, t)\|_{2}^{2}=\|\psi\|_{2}^{2}+\|\phi\|_{2}^{2}=\left(\|F\|_{2}^{2}+\|G\|_{2}^{2}\right) / 2 .
$$

Proof. By Theorem $1, \lim _{t \rightarrow \infty}\|(1-\chi) u(\cdot, t)\|_{2}=0$ and hence

$$
\lim _{t \rightarrow \infty}\|u(\cdot, t)\|_{2}^{2}-\|\chi u(\cdot, t)\|_{2}^{2}=0 .
$$

Thus the corollary follows from the fact (see Brodsky [1]) that

$$
\lim _{t \rightarrow \infty}\|u(\cdot, t)\|_{2}^{2}=\left(\|F\|_{2}^{2}+\|G\|_{2}^{2}\right) / 2 \text {. }
$$

REMARK. Let $V_{n}$ denote the volume of the unit ball in $R^{n}$ so that $\|\chi u(\cdot, t)\|_{2}^{2} \leqq V_{n} t^{n}\|\chi u(\cdot, t)\|_{\infty}^{2}$. Applying the corollary one sees that if $\chi u(\cdot, t) \|_{\infty}=o\left(t^{-n / 2}\right)$ as $t \rightarrow \infty$ then $u=0$, a special case of a result by Littman [3].

The above corollary can be extended to the case where $\chi$ is replaced by the characteristic function of other cones (see Corollary $\left.1^{\prime}\right)$. Theorem 1 will be deduced from Theorem $1^{\prime}$ below. The proof of Theorem $\mathbf{1}^{\prime}$ is based on

\section{Lemma 1. Define}

$$
W_{t}(x)=(2 \pi)^{-n / 2} \int e^{i x \cdot y} \exp \left[-i t \sqrt{ }\left(1+y^{2}\right)\right]\left(1+y^{2}\right)^{-(n+2) / 4} d^{n} y .
$$

Then, for every $f \in L^{1}\left(R^{n}\right)$ and $\lambda \in R^{n}$,

$$
\lim _{t \rightarrow \infty} t^{n / 2} e^{i n \pi / 4} \exp \left[i t / \sqrt{ }\left(1+\lambda^{2}\right)\right] W_{t} * f\left(\frac{\lambda t}{\sqrt{ }\left(1+\lambda^{2}\right)}\right)=\hat{f}(\lambda) .
$$

Proof. In [5] it is shown that for $t>0$,

$$
W_{t}(x)=t^{-n / 2} e^{-i n x / 4} \exp \left[-\sqrt{ }\left(x^{2}-t^{2}\right)\right]+R_{t}(x)
$$

where $\left\|R^{t}\right\|_{\infty}=O\left(t^{-1-n / 2}\right)$ as $t \rightarrow \infty$. By $\sqrt{ }\left(x^{2}-t^{2}\right)$ we mean the value that lies on the positive imaginary axis when $|x|<t$ and on the positive real axis when $|x|>t$. Since $\left\|R_{t}\right\|_{\infty}=O\left(t^{-1-n / 2}\right)$ it is clear that $t^{n / 2}\left\|R_{t} * f\right\|_{\infty}=O\left(t^{-1}\right)$ and hence 


$$
\lim _{t \rightarrow \infty} t^{n / 2} e^{i n \pi / 4} \exp \left[i t \sqrt{ }\left(1+\lambda^{2}\right)\right] R_{t} * f\left(\frac{\lambda t}{\sqrt{ }\left(1+\lambda^{2}\right)}\right)=0 .
$$

Thus to complete the proof of the lemma we must show

$$
\begin{aligned}
& \lim _{t \rightarrow \infty}(2 \pi)^{-n / 2} \int \exp \left(\frac{i t}{\sqrt{ }\left(1+\lambda^{2}\right)}\right. \\
& \left.-\sqrt{ }\left(\left|\frac{\lambda t}{\sqrt{ }\left(1+\lambda^{2}\right)}-x\right|^{2}-t^{2}\right)\right) f(x) d^{n} x \\
& \quad=(2 \pi)^{-n / 2} \int e^{-i \lambda \cdot x} f(x) d^{n} x \equiv \hat{f}(\lambda) .
\end{aligned}
$$

But this is a consequence of Lebesgue's dominated convergence theorem because

$$
\lim _{t \rightarrow \infty} \frac{i t}{\sqrt{ }\left(1+\lambda^{2}\right)}-\sqrt{ }\left(\left|\frac{\lambda t}{\sqrt{ }\left(1+\lambda^{2}\right)}-x\right|^{2}-t^{2}\right)=-i \lambda \cdot x .
$$

For $\phi \in L^{2}\left(R^{n}\right)$ define

$$
U_{t} \phi(x)=(2 \pi)^{-n / 2} \int e^{i x \cdot y} \exp \left[-i t \sqrt{ }\left(1+y^{2}\right)\right] \phi(y) d^{n} y .
$$

CoRollary 2. If there exists $f \in L_{1}\left(R^{n}\right)$ such that $\hat{f}(\lambda)=\left(1+\lambda^{2}\right)^{(n+2) / 4}$ $\cdot \phi(y)$ then

$$
\begin{gathered}
\left\|U_{t} \phi\right\|_{\infty} \leqq(2 \pi)^{-n / 2}\|f\|_{1}\left\|_{1} W_{t}\right\|_{\infty} \sim(2 \pi)^{-n / 2}\|f\|_{1} t^{n / 2} \quad \text { as } t \rightarrow \infty, \\
\lim _{t \rightarrow \infty} t^{n / 2} e^{i \alpha(\lambda, t)} U_{t} \phi\left(\frac{\lambda t}{\sqrt{ }\left(1+\lambda^{2}\right)}\right)=\hat{f}(\lambda)=\left(1+\lambda^{2}\right)^{(n+2) / 4} \phi(\lambda),
\end{gathered}
$$

where $\alpha(\lambda, t) \equiv n \pi / 4+t / \sqrt{ }\left(1+\lambda^{2}\right)$.

Proof. Both (i) and (ii) follow easily from the fact that

$$
\begin{aligned}
U_{t} \phi(x) & =(2 \pi)^{-n / 2} \int e^{i x \cdot \lambda} \exp \left[-i t \sqrt{ }\left(1+\lambda^{2}\right)\right]\left(1+\lambda^{2}\right)^{-(n+2) / 4} \hat{f}(\lambda) d^{n} \lambda \\
& =(2 \pi)^{-n / 2} \int W_{t}(x-y) f(y) d^{n} y \equiv W_{t} * f(x) .
\end{aligned}
$$

REMARK. The proof of Corollary 2 follows the approach used by Brodsky [2] and Segal [7, pp. 95-98] to obtain bounds like that given by (i). Recently, I became aware of a different approach by Littman [4] which when applied to the present situation yields (i) and (ii) with different assumptions on $\phi$. Using Littman's approach Theorem 1 can be extended to more general situations (see [6]). 
Motivated by (ii) of Corollary 2 we define an approximation $A_{t} \phi(x)$ to $U_{t} \phi(x)$ by requiring

$$
t^{n / 2} e^{i \alpha(\lambda, t)} A_{t} \phi\left(\frac{\lambda t}{\sqrt{ }\left(1+\lambda^{2}\right)}\right)=\left(1+\lambda^{2}\right)^{(n+2) / 4} \phi(\lambda), \quad \lambda \in R^{n}, t>0 .
$$

To see how this works out consider the transformation

$$
T_{t}: \lambda \rightarrow x=\frac{\lambda t}{\sqrt{ }\left(1+\lambda^{2}\right)}
$$

that maps $R^{n}$ onto the ball $|x|<t$. Since $x^{2}=\lambda^{2} t^{2} /\left(1+\lambda^{2}\right)$ we have $\lambda^{2}\left(t^{2}-x^{2}\right)=x^{2}$ and hence $T_{t}^{-1}: x \rightarrow \lambda=x / \sqrt{ }\left(t^{2}-x^{2}\right)$. Taking $\lambda=T_{t}^{-1}(x)$ in (4) and multiplying by $t^{-n / 2} e^{-i \alpha}$ gives

$$
\begin{aligned}
A_{t} \phi(x) & =\exp \left[-i \alpha\left(T_{t}^{-1}(x), t\right)\right] t^{-n / 2}\left\{1+\frac{x^{2}}{t^{2}-x^{2}}\right\}^{(n+2) / 4} \phi\left(T_{t}^{-1}(x)\right) \\
& =e^{-i \theta(x, t)} \rho(x, t) \phi\left(\frac{x}{\sqrt{ }\left(t^{2}-x^{2}\right)}\right), \quad|x|<t,
\end{aligned}
$$

where $\theta$ and $\rho$ are the functions defined in Theorem 1 .

Theorem 1'. For $\phi \in L^{2}\left(R^{n}\right)$ define $U_{t} \phi$ and $A_{t} \phi$ by (3) and (5). Then

(i) $\left\|A_{t} \phi\right\|_{2, t}=\|\phi\|_{2}=\left\|U_{t} \phi\right\|_{2}$,

(ii) $\lim _{t \rightarrow \infty}\left\|U_{t} \phi-A_{t} \phi\right\|_{2, t}=0$,

(iii) $\lim _{t \rightarrow \infty} \int_{|x|>t}\left|U_{t} \phi(x)\right|^{2} d x=0$,

where

$$
\|f\|_{2, t} \equiv\left\{\int_{|x|<t}|f(x)|^{2} d^{n} x\right\}^{1 / 2} .
$$

Proof. It is not difficult to check that the Jacobian of $T_{t}$ is

$$
\frac{\partial\left(x_{1}, \cdots, x_{n}\right)}{\partial\left(\lambda_{1}, \cdots, \lambda_{n}\right)}=t^{n}\left(1+\lambda^{2}\right)^{-(n+2) / 2}=\rho^{-2}\left(T_{t}(\lambda), t\right) .
$$

Thus by the change of variable theorem for multiple integrals

$$
\|f\|_{2, t}^{2}=\int\left|f\left(T_{t}(\lambda)\right)\right|^{2} \rho^{-2}\left(T_{t}(\lambda), t\right) d^{n} \lambda .
$$

Taking $f=A_{\iota} \phi$ in (6) we obtain the left-hand side of (i). The other half of (i) is Parseval's equality.

To prove (ii) let $\epsilon>0$ and choose $\phi \in C_{c}^{\infty}\left(R^{n}\right)$ such that $\|\phi-\phi\|_{2}$ $<\epsilon / 3$. Applying (i) to $\phi-\delta$ we have 


$$
\left\|U_{t} \phi-U_{t} \tilde{\phi}\right\|_{2, t} \leqq\left\|U_{t} \phi-U_{t} \tilde{\phi}\right\|_{2}=\left\|A_{t} \phi-A_{t} \tilde{\phi}\right\|_{2, t}=\|\phi-\tilde{\phi}\|_{2} .
$$

Thus

$$
\begin{aligned}
\left\|U_{\iota} \phi-A_{\imath} \phi\right\|_{2, t} & \leqq\left\|U_{\iota} \phi-U_{t} \tilde{\phi}\right\|_{2, t}+\left\|U_{\iota} \tilde{\phi}-A_{\imath} \tilde{\phi}\right\|_{2, t}+\left\|A_{\iota} \tilde{\phi}-A_{t} \phi\right\|_{2, \imath} \\
& <\epsilon / 3+\left\|U_{\imath} \tilde{\phi}-A_{\imath} \tilde{\phi}\right\|_{2, t}+\epsilon / 3
\end{aligned}
$$

so to prove (ii) we need only show there exists $\tau$ such that $t>\tau$ implies $\left\|U_{t} \tilde{\phi}-A_{t} \tilde{\phi}\right\|_{2, t}<\epsilon / 3$. Since this amounts to proving (ii) with $\phi$ replaced by $\tilde{\phi}$, we simply assume $\phi \in C_{c}^{\infty}\left(R^{n}\right)$.

Applying (6) we have

$$
\begin{aligned}
&\left\|U_{t} \phi-A_{t} \phi\right\|_{2, t}^{2}=\int\left|U_{t} \phi\left(\frac{\lambda t}{\sqrt{ }\left(1+\lambda^{2}\right)}\right)-A_{t} \phi\left(\frac{\lambda t}{\sqrt{ }\left(1+\lambda^{2}\right)}\right)\right|^{2} \\
&=\int \mid \rho^{-2}\left(T_{t}(\lambda), t\right) d^{n} \lambda \\
&\left.e^{-i \alpha(\lambda, t)}\left[g_{t}(\lambda)-\phi(\lambda)\right]\right|^{2} d^{n} \lambda
\end{aligned}
$$

where

$$
g_{t}(\lambda)=e^{i \alpha(\lambda, t)} t^{n / 2}\left(1+\lambda^{2}\right)^{(n+2) / 4} U_{t} \phi\left(\frac{\lambda t}{\sqrt{ }\left(1+\lambda^{2}\right)}\right) .
$$

Clearly $\left\|U_{t} \phi\right\|_{2, t}^{2}=\int\left|g_{t}(\lambda)\right|^{2} d^{n} \lambda$ and hence

$$
\left\|g_{t}\right\|_{2} \leqq\left\|U_{t} \phi\right\|_{2}=\|\phi\|_{2} \text {. }
$$

Since $\phi \in C_{c}^{\infty}\left(R^{n}\right)$ part (ii) of Corollary 2 can be used to conclude that for every $\lambda \in R^{n}$

$$
\lim _{t \rightarrow \infty} g_{t}(\lambda)=\phi(\lambda) .
$$

An application of Fatou's lemma and Egoroff's theorem shows that (8) and (9) imply $\lim _{t \rightarrow \infty}\left\|g_{t}-\phi\right\|_{2}=0$ which in view of (7) establishes (ii).

To prove (iii) we must show $\lim _{t \rightarrow 0}\left\|U_{t} \phi\right\|_{2}^{2}-\left\|U_{t} \phi\right\|_{2, t}^{2}=0$ which is obvious from (i) and (ii).

Proof of Theorem 1. Since

$$
u(x, t)=\overline{U_{\imath} \Psi(-x)}+U_{\imath} \phi(x), \quad a(x, t)=\overline{A_{\imath} \Psi(-x)}+A_{\imath} \phi(x),
$$

Theorem 1 follows immediately from Theorem $1^{\prime}$.

Let $\Gamma$ be a cone inside the cone $\{(x, t):|x|<t\}$. That is, assume $(x, t) \in \Gamma$ implies $|x|<t$ and $(s x, s t) \in \Gamma$ for all $s>0$. Put 


$$
B=\left\{\frac{x}{\sqrt{ }\left(1-x^{2}\right)}:(x, 1) \in \Gamma\right\}
$$

and let $\beta(\lambda)$ be the characteristic function of $B$. Then the characteristic function $\gamma(x, t)$ of $\Gamma$ is zero for $|x|>t$ and satisfies

$$
\gamma(x, t)=\beta \circ T_{t}^{-1}(x)=\beta\left(\frac{x}{\sqrt{ }\left(t^{2}-x^{2}\right)}\right) \text { for }|x|<t .
$$

Corollary $1^{\prime}$. Let $\gamma(x, t), B$ be as above and let $u(x, t), \phi(y), \psi(y)$ be as in Theorem 1. Then

$$
\lim _{t \rightarrow \infty}\|\gamma u(\cdot, t)\|_{2}^{2}=\int_{B}|\psi(-\lambda)|^{2}+|\phi(\lambda)|^{2} d^{n} \lambda .
$$

REMARK. Similar expressions can be obtained for the kinetic and potential energies $\left\|\gamma u_{t}\right\|_{2}^{2},\|\gamma u\|_{2}^{2}+\sum\left\|\gamma u_{x_{i}}\right\|_{2}^{2}$ since $u_{t}$ and $u_{x_{i}}$ can also be written in the form of equation (1). This gives an extension of the Virial theorem stated in Brodsky [1].

Proof. By Theorem 1 it suffices to prove the corollary with $u(x, t)$ replaced by $a(x, t)$. When $|x|<t$ we have

$$
\begin{aligned}
\gamma(x, t) a(x, t)=\beta\left(\frac{x}{\sqrt{ }\left(t^{2}-x^{2}\right)}\right)\left\{e^{i \theta(x, t)} \psi\right. & \left(\frac{-x}{\sqrt{ }\left(t^{2}-x^{2}\right)}\right) \\
& \left.+e^{-i \theta(x, t)} \phi\left(\frac{x}{\sqrt{ }\left(t^{2}-x^{2}\right)}\right)\right\} \rho(x, t),
\end{aligned}
$$

and hence, by (6),

$$
\|\gamma a(\cdot, t)\|_{2, t}^{2}=\int \beta(\lambda)\left|e^{i \alpha(\lambda, t)} \psi(-\lambda)+e^{-i \alpha(\lambda, t)} \phi(\lambda)\right|^{2} d^{n} \lambda .
$$

Since $\left|e^{i a} \psi+e^{-i \alpha} \phi\right|^{2}=|\psi|^{2}+|\phi|^{2}+e^{2 i \alpha} \psi \phi+e^{-2 i \alpha} \bar{\psi} \phi$ the corollary follows from

Lemma 2. For $f \in L^{1}\left(R^{n}\right)$ define $I_{f}(t)=\int \exp \left[i t / \sqrt{ }\left(1+\lambda^{2}\right)\right] f(\lambda) d^{n} \lambda$. Then $\lim _{|\imath| \rightarrow \infty} I_{f}(t)=0$.

Proof. Since $C_{c}\left(R^{n}\right)$ is dense in $L^{1}\left(R^{n}\right)$ and since $\left|I_{f}(t)-I_{\sigma}(t)\right|$ $\leqq\|f-g\|_{1}$ it suffices to prove the lemma when $f$ is continuous and has compact support. Switching to spherical coordinates gives

$$
I_{f}(t)=\int_{0}^{\infty} \exp \left[i t / \sqrt{ }\left(1+r^{2}\right)\right] F(r) d r, \quad F(r)=r^{n-1} \int_{|y|=1} f(r y) d S(y) .
$$

Applying the change of variable $u=1 / \sqrt{ }\left(1+r^{2}\right)$ yields 


$$
I_{f}(t)=\int_{0}^{1} e^{i t u} \phi(u) d u, \quad \phi(u)=F\left(\frac{\sqrt{ }\left(1-u^{2}\right)}{u}\right) \frac{1}{u^{2} \sqrt{ }\left(1-u^{2}\right)} .
$$

Since $\sqrt{ }\left(1-u^{2}\right) \phi(u) \in C_{c}((0,1])$, the lemma follows from the Riemann-Lebesgue theorem.

REMARK ON $L^{p}$ BEHAVIOR. By Hölder's inequality

$$
\begin{aligned}
\|\gamma u(\cdot, t)\|_{2}^{2} & \leqq\|\gamma(\cdot, t)\|_{q}\left\|\gamma u^{2}(\cdot, t)\right\|_{q^{\prime}} \\
& =\|\gamma(\cdot, t)\|_{1}^{1 / q}\|\gamma u(\cdot, t)\|_{2 q^{\prime}}^{2} \\
& =\left(\|\gamma(\cdot, 1)\|_{1} t^{n}\right)^{(1-2 / p)}\|\gamma u(\cdot, t)\|_{p}^{2}
\end{aligned}
$$

where $1-1 / q=1 / q^{\prime}=2 / p$. Thus Corollary $1^{\prime}$ gives a lower bound for $\lim \inf _{t \rightarrow \infty} t^{n / 2} t^{-n / p}\|\gamma u(\cdot, t)\|_{p}, p \geqq 2$, which is positive unless $\phi(\lambda)$ and $\psi(-\lambda)$ vanish for $\lambda \in B$.

On the other hand, by using Corollary 2(i) and the estimate $\|f\|_{p}^{p} \leqq\|f\|_{2}^{2}\|f\|_{\infty}^{p-2}, 2 \leqq p<\infty$ one can show

$$
\|u(\cdot, t)\|_{p}=O\left(t^{-n / 2} t^{n / p}\right), \quad\|u(\cdot, t)-a(\cdot, t)\|_{p}=o\left(t^{n / 2} t^{n / p}\right)
$$

as $t \rightarrow \infty$, provided $\phi$ and $\psi$ satisfy the condition of Corollary 2 .

\section{REFERENCES}

1. A. R. Brodsky, On the asymptotic behavior of solutions of the wave equations, Proc. Amer. Math. Soc. 18 (1967), 207-208. MR 35 \#3289.

2. - Asymptotic decay of solutions to the relativistic wave equation, Thesis, M.I.T., Cambridge, Mass., 1964.

3. W. Littman, Maximal rates of decay of solutions of partial differential equations, Bull. Amer. Math. Soc. 75 (1969), 1273-1275.

4. - Fourier transforms of surface-carried measures and differentiability of surface averages, Bull. Amer. Math. Soc. 69 (1963), 766-770. MR 27 \#5086.

5. S. Nelson, On some solutions to the Klein-Gordon equation related to an integral of Sonine, Trans. Amer. Math. Soc. (to appear).

6. - $L^{2}$ asymptotes for Fourier transforms of surface-carried measures, Proc. Amer. Math. Soc. (to appear).

7. I. E. Segal, Quantization and dispersion for non-linear relativistic equations, Proc. Conference Mathematical Theory of Elementary Particles (Dedham, Mass., 1965), M.I.T. Press, Cambridge, Mass., 1966, pp. 79-108. MR 36 \#542.

Iowa State University, Ames, Iowa 50010 